\title{
Applying high-throughput computational techniques for discovering next-generation of permanent magnets
}

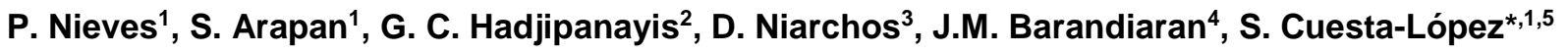 \\ ${ }^{1}$ ICCRAM, International research Center in Critical Raw Materials and Advanced Industrial Technologies, University of Burgos, \\ 09001, Burgos, Spain. \\ ${ }^{2}$ Department of Physics and Astronomy, University of Delaware, Newark, DE, USA. \\ ${ }^{3}$ Institute of Nanoscience and Nanotechnology, NCSR Demokritos, Athens 15310, Greece \\ ${ }^{4}$ BCMaterials, Ibaizabal Bidea, Bdng. 500, Parque Cientıfico y Tecnologico de Bizkaia, 48160 Derio, Spain \\ ${ }^{5}$ Advanced Materials, Nuclear Technology and NanoBioTechnology Dept. University of Burgos, 09001, Burgos, Spain.
}

Received ZZZ, revised ZZZ, accepted ZZZ

Published online ZZZ (Dates will be provided by the publisher.)

Keywords Magnetism, magnetic materials, permanent magnets, genome materials, high-throughput computation

* Corresponding author: e-mail scuesta@ubu.es Phone: +34 947259062

The uncertainty in rare-earth market resulted in worldwide efforts to develop rare-earth-lean/free permanent magnets. In this paper we discuss about this problem and analyse how advances in computational and theoretical condensed matter physics could be essential in the development of a new generation of high-performance permanent magnets via high-throughput computational technique for material design. Additionally, we show that an adaptive genetic algorithm based methodology could be a useful tool for finding new magnetic phases. In particular, we apply such approach to $\mathrm{Fe}_{0.75} \mathrm{Sn}_{0.25}$ compound recovering well-known experimental results and also finding new low-energy magnetic metastable structures.

1 Introduction to the critical raw material problem in permanent magnets During last century the intense research on magnetic materials led to a continuous increase of permanent magnets (PM) performance [1]. These advances have allowed the development of a large number of industrial applications of PM, including the electric, electronic and automobile industries, communications, information technologies and automatic control engineering [2]. Nowadays, PM are widely used in many applications in our society, we can find from cheap ferrites with moderate performance used in decorative or holding objects on refrigerator doors, loudspeakers, small motors, actuators, electronic components, ... to high performance

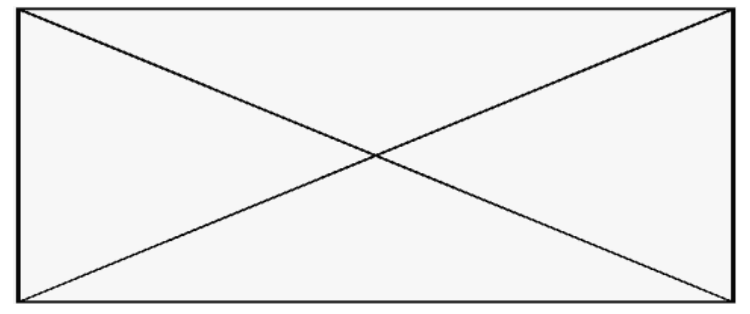

This is the caption of the optional abstract figure. If there is no figure here, the abstract text should be divided into both columns. and expensive Rare-Earth (RE) magnets like $\mathrm{NdFeB}$ and SmCo used in large motors, generators, magnetic resonance imaging equipment, metallic elements separators, electron tubes, oil dewaxing, ...

The main function of PM is to create a magnetic field $\mathrm{H}$ in an air gap. This field is proportional to the volume of the magnet and the stored energy product $\mathrm{B}_{\mathrm{m}} \mathrm{H}_{\mathrm{m}}$, hence, magnets with high $\mathrm{B}_{\mathrm{m}} \mathrm{H}_{\mathrm{m}}$ are highly desirable. In fact, the maximum energy product $(\mathrm{BH})_{\max }$ that a PM can provide is considered as figure of merit of it [3]. Another important properties of a PM are the remanence $\mathrm{M}_{\mathrm{r}}$, coercivity $\mathrm{H}_{\mathrm{c}}$ and Curie temperature $\mathrm{T}_{\mathrm{C}}$. The remanence determines the max- 
imum field $\mathrm{H}$ within the air gap, while the coercivity determines the 'resistance' against demagnetisation by external magnetic fields. High values of $\mathrm{H}_{\mathrm{c}}$ require high magnetic anisotropy whose origin may be magnetocrystalline(created by the competition between spin-orbit coupling and crystal field) or shape-related (originated by magnetic moment dipole fields). The Curie temperature, where above it a ferromagnetic material becomes paramagnetic, is also very important since it stablishes the temperature range in which the PM can be used in an application. $\mathrm{T}_{\mathrm{C}}$ is related to the microscopic exchange interaction between neighbour atoms. Notice that maximum energy product density, coercivity and remanence are temperature dependent and they typically decrease as temperature increases, therefore the performance of a PM may diminish rapidly with increasing temperature. Finally, another important fact in PM design is the resistance to corrosion, oxidation and decomposition. In Table 1 we show the remanence, coercivity and maximum energy product of some representative PM.

Table 1 Properties of some representative permanent magnets $[1,9]$.

\begin{tabular}{|c|c|c|c|c|}
\hline Compound & Ref. & $\begin{array}{c}\mu_{0} \mathrm{M}_{\mathrm{r}} \\
(\mathrm{T})\end{array}$ & $\begin{array}{c}\mu_{0} \mathrm{H}_{\mathrm{c}} \\
(\mathrm{T})\end{array}$ & $\begin{array}{l}(\mathrm{BH})_{\max } \\
\left(\mathrm{kJ} / \mathrm{m}^{3}\right)\end{array}$ \\
\hline $\begin{array}{l}\text { Co-Steel } \\
\text { Alnico } 5\end{array}$ & $\begin{array}{l}{[1]} \\
{[1]}\end{array}$ & $\begin{array}{l}1.07 \\
1.05\end{array}$ & $\begin{array}{l}0.02 \\
0.06\end{array}$ & $\begin{array}{c}6 \\
44\end{array}$ \\
\hline $\mathrm{BaFe}_{12} \mathrm{O}_{19}$ & {$[1]$} & 0.42 & 0.31 & 34 \\
\hline $\begin{array}{c}\mathrm{SmCo}_{5} \\
\text { (metal-bonded) }\end{array}$ & [9] & 0.92 & 1.88 & 175 \\
\hline $\begin{array}{l}\mathrm{Nd}_{2} \mathrm{Fe}_{14} \mathrm{~B} \\
\text { (sintered) }\end{array}$ & [9] & $1.0-1.4$ & $1.0-2.5$ & $200-440$ \\
\hline
\end{tabular}

In the beginning of last century, high carbon steel with a maximum energy product of 1 MGOe was the highest performance PM. After 30-year period from 1932 of Alnicos (heat-treated $\mathrm{Fe}-\mathrm{Co}-\mathrm{Ni}-\mathrm{Al}$ alloys) development, the maximum energy product increased by a factor of 10 [1]. Alnicos have some interesting properties as high remanence and $\mathrm{T}_{\mathrm{C}}$. Unfortunately, its coercivity is very low due to an inefficient microstructure, which favours the magnetization reversal [4]. At the present time, the research in this $\mathrm{PM}$ is focused on improving the coercivity by reducing the scale of the microstructure, which could improve anisotropy, and reducing the interaction strength between FeCorich precipitates, which could enhance the domain wall pinning [5,6]. During Alnicos development period, cheap iron oxide magnets called Ferrites where also discovered, which exhibit a higher coercivity but lower maximum energy product ( $\sim 5 \mathrm{MGOe}$ ) than Alnico magnets. For example, Ferrites like $\mathrm{MFe}_{12} \mathrm{O}_{19}$, where $\mathrm{M}$ is $\mathrm{Sr}$ or $\mathrm{Ba}$, have high coercivity, high resistant to corrosion and low cost. However, the antiferromagetic coupling between $\mathrm{Fe}$ atoms leads to a very low remanence. Nowadays, some efforts are aimed at reducing the magnetization of one sublattice without changing too much $\mathrm{T}_{\mathrm{C}}$. Next great revolution in PM was achieved with the introduction of RE magnets in 1969, in particular with $\mathrm{Sm}_{2} \mathrm{Co}_{17}$ and $\mathrm{SmCo}_{5}$, achieving a maximum energy product of 18-20 MGOe. However, Co price increment in the late 1970s made research focus on the search of Co-free iron-based PM, leading to the discovery of $\mathrm{Nd}_{2} \mathrm{Fe}_{14} \mathrm{~B}$ magnets in 1983 , which not only reduced costs but also increased energy product density (see Fig. 1). Unfortunately, in the last three decades only small improvements of the energy product has been achieved, where $\mathrm{NdFeB}$ compound, with a maximum energy product of $56 \mathrm{MGOe}$, is still the highest-performance PM that we have today. Fig. 2 shows the crystal structure of $\mathrm{Nd}_{2} \mathrm{Fe}_{14} \mathrm{~B}$ and Ferrite $\mathrm{BaFe}_{12} \mathrm{O}_{19}$. The key of these RE-PM is the good balance between the high magnetocrystalline anisotropy provided by the large spin-orbit coupling of the REelements (leading to a high coercivity) and large saturation magnetization given by the transition metal element. As a result, current high-performance PM are totally dependent on not abundant and expensive RE materials like $\mathrm{Nd}, \mathrm{Sm}$ and Dy (Dy is frequently used for enhancing the performance of $\mathrm{NdFeB}$ magnets at high temperatures), which have now become critical raw materials. This worrying situation makes the development of RE-free PM an urgent priority but also a great scientific and technological challenge [7]. Advances and current research lines in PM that don't contain RE elements can be found in Refs. $[6,8,9]$.

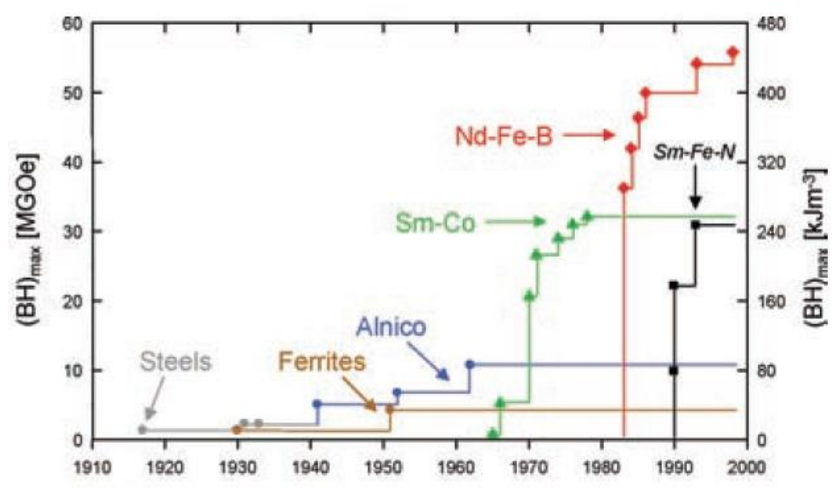

Figure 1 Historical development of maximum energy product of PM [2]. 
(a)

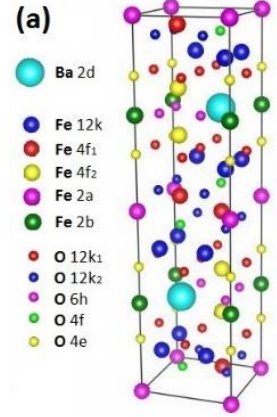

(b)

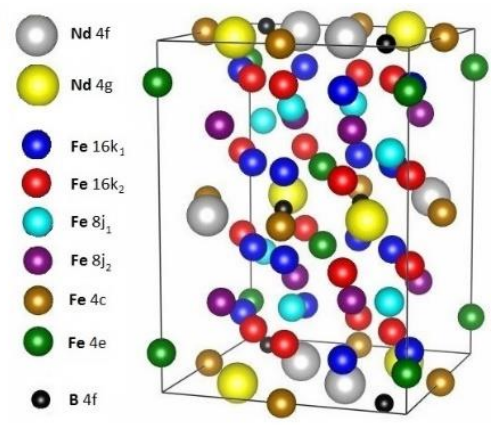

Figure 2 (a) Crystal structure of Ferrite $\mathrm{BaFe}_{12} \mathrm{O}_{19}$. (b) Crystal structure of $\mathrm{Nd}_{2} \mathrm{Fe}_{14} \mathrm{~B}$.

As in the late 1970s with Co-PM crisis, which curiously led to RE-PM discovery, now we are in a similar situation with RE-PM crisis. The USA became the primary producer of RE-oxides until in the mid-1980s China entered the world market. Gradually, since the beginning of the 1990s, China has been dominating the market to an increasing extent by means of its very low production costs and large reserves. Today, there is a Chinese quasimonopoly of RE-oxides market. As it is shown in Fig.3a the world production of RE-oxides, specially $\mathrm{La}, \mathrm{Ce}$ and $\mathrm{Nd}$, has increased in the last years due to the large demand of different industries. In 2008 around $20 \%$ of the production of RE-oxides were consumed by the PM industry [10]. Rare earth prices generally peaked in 2011 (see Fig.3b), this anomalous behaviour could be due to speculation and hoarding of RE-oxides, the subsequent decreases after 2011 may also be due to sell-offs by the speculators [11]. Clearly, this unstable market situation harms all REdependent industries, as the PM industry, and makes them search for other viable alternatives.
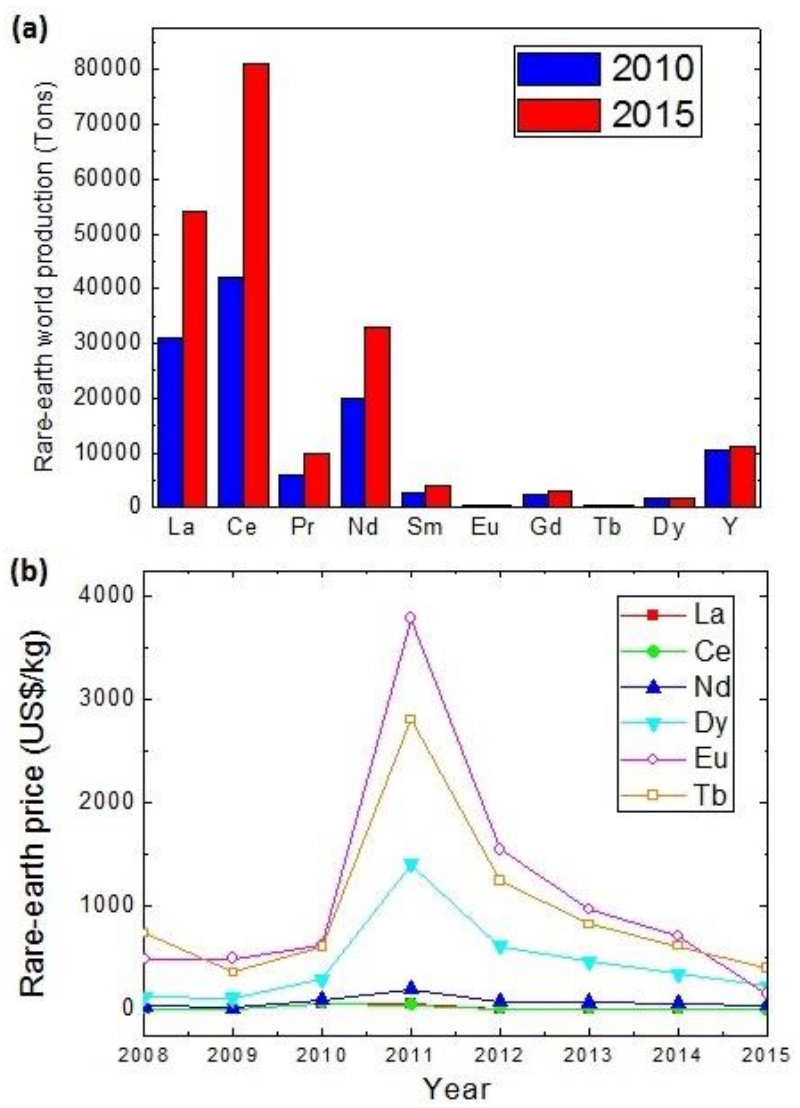

Figure 3 (a) Estimated rare-earth oxide world production in 2010 and 2015 [11]. (b) Price evolution of some rare-earth oxides $[12,13]$.

In order to solve this problem most efforts are focused on reducing and recycling the content of these RE elements in PM. For reducing RE content of RE-PM the following strategies have been used: i) large shape anisotropy, with non-spherical particles, to compensate for the loss in magnetocrystalline anisotropy when reducing RE content, ii) substitution of Dy by a more abundant and cheaper RE as $\mathrm{Pr}$ and $\mathrm{Ce}$, iii) reducing Dy content, by diffusion of some Dy along the grain boundaries for its introduction only at places required for coercivity enhancement. Both for economic and environmental reasons the recycling of REbased magnets has gained increasing attention and importance in the permanent magnet industry. In our modern society, the number of obsolete devices which contain RE$\mathrm{PM}$ is increasing very rapidly, which may provide a cheap source of RE-PM. For example, it was estimated [14,15] that the hard disk drive (HDD) industry could source around $57-64 \%$ of its $\mathrm{NdFeB}$ requirement from recycled HDD sources, which equates to approximately $3-11 \%$ of total NdFeB demand. Recently, it has been shown that hydrogen can be used as a very effective processing agent to decrepitate sintered $\mathrm{NdFeB}$ magnets from HDDs using a method called Hydrogen Processing of Magnetic Scrap 
(HPMS), this technique may also be applied to other devices such as electric motors, generators and actuators [16]. At the present time, Hitachi recycles RE-PM using a selfdeveloped process that separates the rare-earth magnets from HDD and air conditioner compressors [17].

So far the historical development of PM shows that experimental approaches were the most successful way for finding new and better PM. However, this traditional trend may reverse due to theoretical advances in materials science and powerful computational tools that are available at the present time, which could be essential in the discovery of the so desired next-generation of PM.

The aim of this paper is to briefly present the problematic of the dependence on critical raw materials by the technology underlying permanent magnets, at the same time that giving an introductory review to the main computational existing alternatives for the discovery of new magnetic materials. In section 2 we shortly describe some standard theoretical methods widely used for studying magnetism in materials, while in section 3 we present current novel high-throughput computational techniques as a promising approach for the discovery of new magnetic phases, thus contributing to find high-performance RE-free PM. Finally, in section 4 we discuss a particular genetic adaptive algorithm based methodology as a high throughput screening approach in the quest of new permanent magnets. Such a methodology has been preliminary validated thanks to first calculations on the crystal structure from an experimentally well-known compound: $\mathrm{Fe}_{0.75} \mathrm{Sn}_{0.25}$.

2 Common theoretical approaches to permanent magnets Short (exchange interaction, spin-orbit coupling, ...) and long (dipole-dipole fields, ...) range interactions in magnetic materials make difficult the study of them. A complete description of their behaviour involves very different spatial scales going from anstrong (like microscopic interactions at atomic level) to macroscale (where magnetic domains form, usually with micrometer sizes). In the last decades, theoretical methods have been extensively developed for studying these materials. This fact has opened the possibility of predict and design PM very accurately.

One of the most successful theoretical approaches to condensed mater physics is Density Functional Theory (DFT). It has had a great impact on fundamental and industrial research, including mechanical properties and structural materials, catalysis and surface physics, nanotechnology, biomaterials, magnetism and magnetic materials [18] Here, we present some basic ideas behind this theory and how some intrinsic properties of PM can be calculated with it, a detailed modern introduction to DFT can be found in Ref. [19].
One way to deal with a many-body problem is to assume that each electron can be considered separately, moving in a mean field potential. This potential models the effects of all the other particles in the system, as well as any external potential acting on the system. In 1965 Kohn and Sham suggested to consider a non-interacting $n$-electron system $\Psi_{\mathrm{i}}(\boldsymbol{r})$, which has the same charge density as the real one. Thus, the complex many-body problem reduces to a set of one-electron Kohn-Sham equations:

$$
\left[-\frac{\hbar}{2 m_{i}} \nabla^{2}+V_{K s}(\boldsymbol{r})\right] \Psi_{i}(\boldsymbol{r})=\varepsilon_{i} \Psi_{i}(\boldsymbol{r}){ }
$$

where $\varepsilon_{i}$ is the eigenvalue associated to the single-particle state $\Psi_{i}(\boldsymbol{r})$ and $V_{K g}(\boldsymbol{r})$ is an effective potential which contains the electrons and nuclei Coulomb potential and the so-called exchange-correlation potential. In 1972 von Barth and Hedin and in 1973 Rajagopal and Callaway generalized DFT to include the spin polarization, allowing to an accurate description of magnetism and magnetic materials [20,21]. In standard DFT methods the exchangecorrelation potential is calculated via the local density approximation (LSDA) or in a more advance way through the generalized gradient approximation (GGA). These DFT approaches give reasonable results of important intrinsic properties of PM at ground state as the saturation magnetization, magnetocrystalline anisotropy and exchange integrals. For example, the magnetization due to electron spins (spin moments) in a collinear calculation reads

$$
M_{\text {spin }}=\mu_{B} \int d \boldsymbol{r}\left[n^{\mathbb{}}(\boldsymbol{r})-n^{\Downarrow}(\boldsymbol{r})\right]_{x}
$$

where $\mu_{E}$ is the Bohr magneton, $n^{\widetilde{ }}$ and $n^{\Downarrow}$ are the spin-up and spin-down part of the electron density (density of states, DOS), respectively, which are obtained solving Eq. (1) self-consistently. In Table 2 we present a comparison of $\mathrm{M}_{\text {spin }}$ calculated with DFT and experimentally for Fe, Co and $\mathrm{Ni}$, showing good agreement. More complex cases as non-collinear systems or orbital magnetism can also be treated with DFT [20,21]. For calculating the magnetocrystalline anisotropy the spin-orbit coupling interaction $(\propto \widehat{\boldsymbol{S}} \cdot \widehat{\boldsymbol{L}}$, where $\widehat{\boldsymbol{S}}$ and $\widehat{\boldsymbol{L}}$ are the electron spin and angular momentum operators, respectively) must be taken into account [22]. Typically, the calculation of magnetocrystalline anisotropy in PM requires simulations with high precision due to the quite small energy dependence on the magnetization orientation $(\sim \mu \mathrm{eV}$, see Table 2$)$. Regarding to exchange integrals, one successful DFT method for determine them in PM is through the two-step approach suggested by Lichtenstein et al. [23]. Here, an initial selfconsistent electronic structure calculation is performed for a collinear spin structure at zero temperature, then exchange integrals are determined using the one-electron Greens function as $[23,24]$

$\mathrm{I}_{\mathrm{RR}}=\frac{1}{\pi} \operatorname{Im} \int_{-\mathrm{m}}^{E_{\mathrm{F}}} d E \int_{\Omega_{\mathrm{R}}} d \boldsymbol{r} \int_{\Omega_{\mathrm{R}^{\prime}}} d \boldsymbol{r}^{\prime} B_{\mathrm{EX}}(\boldsymbol{r}) G_{+}^{\uparrow} B_{\mathrm{Ex}}\left(\boldsymbol{r}^{\prime}\right) G_{-}^{\downarrow},(3)$ 


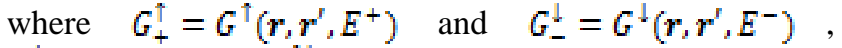
$E^{ \pm}=\lim _{-} E \pm \alpha, G^{\top \downarrow}$ are spin-dependent one electron retarded Greens functions, $E_{F}$ is the Fermi energy, $\Omega_{\mathrm{R}}$ is the atomic cell at lattice site $\mathbf{R}$ and $B_{\sigma x}$ is the magnetic field from the exchange-correlation potential. Once the exchange parameters are obtained, one can make an estimation of $\mathrm{T}_{\mathrm{C}}$ either within the mean-field approximation (MFA) or through the random-phase approximation (RPA) as

$$
T_{C}^{M P A}=\frac{2}{a k_{B}} J(0), T_{C}^{R P A}=\frac{2 N}{a k_{B}}\left[\Sigma_{q} \frac{1}{j(0)-f(q)}\right]^{-1},
$$

with $7(\boldsymbol{q})=\Sigma_{\mathrm{R}} J_{0 \mathrm{R}} \exp (i \boldsymbol{q} \cdot \boldsymbol{R}), \boldsymbol{f}(0)=\Sigma_{\mathrm{R}} J_{\mathrm{oR}}, \boldsymbol{q}$ denotes a vector in the Brillouin zone of the lattice, $N$ is the number of q-vectors and $k_{B}$ is the Boltzmann constant. Calculations of $\mathrm{T}_{\mathrm{C}}$ using DFT and experimentally are shown in Table 2. We see that RPA gives better results than MFA for $\mathrm{Fe}$ and $\mathrm{Co}$, but not for Ni. Alternatively, $\mathrm{T}_{\mathrm{C}}$ can also be calculated through Monte Carlo technique [25] and spin dynamics [26] with reliable results.

Table 2 Calculated and experimental properties for $\mathrm{Fe}$, Co and $\mathrm{Ni} . \Delta \mathrm{E}$ is the energy difference between states with total magnetization pointing along (001) and (111)-directions, that is, $\Delta \mathrm{E}=\mathrm{E}(001)-\mathrm{E}(111)$.

\begin{tabular}{cccccc}
\hline \multicolumn{1}{c}{ Property } & Source & Ref. & Fe (bcc) & Co (fcc) & $\mathrm{Ni}(\mathrm{fcc})$ \\
\hline $\mathrm{M}_{\text {spin }}\left(\mu_{\mathrm{B}} /\right.$ atom) & DFT (LSDA) & {$[21]$} & 2.15 & 1.56 & 0.59 \\
$\mathrm{M}_{\text {spin }}\left(\mu_{\mathrm{B}} /\right.$ atom) & DFT (GGA) & {$[21]$} & 2.22 & 1.62 & 0.62 \\
$\mathrm{M}_{\text {spin }}\left(\mu_{\mathrm{B}} /\right.$ atom) & Experiment & {$[21]$} & 2.12 & 1.57 & 0.55 \\
$\Delta \mathrm{E}(\mu \mathrm{eV})$ & DFT & {$[20]$} & -2.6 & 2.4 & 1.0 \\
$\Delta \mathrm{E}(\mu \mathrm{eV})$ & Experiment & {$[20]$} & -1.34 & 1.66 & 2.7 \\
$\mathrm{~T}_{\mathrm{C}}(\mathrm{K})$ & DFT+MFA & {$[27]$} & 1414 & 1645 & 397 \\
$\mathrm{~T}_{\mathrm{C}}(\mathrm{K})$ & DFT+RPA & {$[27]$} & 950 & 1311 & 350 \\
$\mathrm{~T}_{\mathrm{C}}(\mathrm{K})$ & Experiment & {$[27]$} & 1044 & 1388 & 624 \\
\hline
\end{tabular}

Unfortunately, PM are so complex that a complete description of them cannot be achieved only from first principle techniques. In order to include temperature and long range interactions effects properly one must combine DFT with other approaches in what is called multiscale modelling. Namely, ab-initio calculations (the most widely used formalism is DFT) are initially used to calculate lattice structure and intrinsic magnetic parameters as atomic magnetic moment, exchange integrals, magnetocrystalline anisotropy, etc.... DFT can also be used to derive interatomic potentials which serve as input for classical molecular dynamic simulations for the calculation of atom positions at materials interfaces. Then these parameters can be used in atomistic classical (Heisenberg-like) models [28] where the temperature dependence of the equilibrium magnetization $\mathrm{Ms}(\mathrm{T})$, anisotropy $\mathrm{K}(\mathrm{T})$ and exchange stiffness $\mathrm{A}(\mathrm{T})$, among other properties like $\mathrm{T}_{\mathrm{C}}$ can be calculated. At this stage thermodynamic models can be applied in order to compute the finite temperature magnetic phase diagram, which gives guidelines for the required composition of a magnet. Finally, the temperature dependence of these parameters is included in mesoscopic models as micromagnetics [3], based on the continuum approximation, which can describe the magnetic behaviour at large spatial scale (including the magnet shape and its microstructure) and calculate magnetic extrinsic properties as hysteresis loops, coercivity, remanence and energy product. Examples of the application of this methodology to different magnetic alloys like FePt or Permalloy can be found in Refs. [29,30].

\section{High-throughput computational techniques} for permanent magnet design The high-throughput (HT) computational technique is defined as an automatic throughput of large amount of data [31]. Although the ultimate goal of a HT method is to provide new materials with desired properties and functionality, the throughput of data is not limited only to the combinatorial evaluation of materials properties. Within the past decade we have witnessed an increasing impact of computer simulations on materials science. Basic elements of any material are interacting electrons and nuclei, and the solution of quantum mechanical equations is necessary for the accurate description of any system of these interacting particles. The development of the DFT marks a breakthrough in the field of accurate and efficient numerical techniques of solving quantum mechanical equations. DFT methods have been implemented in increasingly sophisticated codes boosting the theoretical research on materials across physics, chemistry, materials science, biology, and geophysics. Modern DFT codes became very robust, enabling to perform calculations in an automated way, and, thus, opening the way to HT computation. DFT methods provide, usually, the properties of the ground state of the system, and for most of industrial applications one is interested in properties at finite temperature. Therefore, for a computational materials design, thermodynamic simulations follow quantum mechanical calculations. In this way, one may generate a huge database of thermodynamic and electronic properties of known structures and then use data mining techniques to search for materials with desired properties. Thus the first step in the implementation of a computational HT is the accurate numerical simulation of materials properties by a combined DFT and thermodynamic approach.

The next important stage of the HT technique is the rational storage of materials data. One has to devise a way for systematic storage of information obtained from calculations and efficient access of this information from database repositories. Examples of such databases are the AFLOWLIB.org [32] and the Materials Project.org [33]. Relational databases offer the possibility to store data compactly and retrieve it efficiently. Providing a welldesigned interface for the data allows exploring intuitively a large amount of data, and enhances the probability of 
Author, Author, and Author: Short title

finding new compounds with desired properties. An important detail of the database architecture is the identification of the relevant data for storage.

Finally, the third step in a HT scheme is extraction of information and selection of materials of interest. This step is the most challenging one since it requires a deep understanding of the physical problem. The intelligent data mining search is performed by using appropriate descriptors, which connect the microscopic data gained from calculations (like, for example, formation energies, magnetic moments) with macroscopic properties of materials (experimentally measured quantities). Thus an effective HT implementation requires identification of suitable descriptors.

One important aspect of a computational HT materials design compared to the HT experimental approach is the ability to augment the database of existing materials with a large amount of hypothetical materials, which experimental discovery is bound by time-consuming procedure of synthesis or high production cost. Quantum mechanical and thermodynamic calculations allow a virtual synthesis of various materials, and, thus, enhancing the possibility to discover new materials with desired functionality. This approach, however, requires an additional step of experimental validation of predicted structures. In this respect, a proper descriptor to describe the stability of new materials would be the formation enthalpy. Computational schemes like evolutionary and genetic algorithms [34], cluster expansion optimization [35] and structure map analysis [36], which evaluate the descriptors in order to guide new calculations, have been devised.

Recently, DFT techniques have been used for high throughput screening (HTS) in rare-earth based systems for materials design [37, 31]. Another HTS investigation has been performed where full Heusler alloys were screened with common $\mathrm{X} 2 \mathrm{YZ}$ ordered structure as well as in the inverse ordered $(\mathrm{XY}) \mathrm{XZ}$ phase in view of their thermal stability and the existence of a tetragonal ground state [38]. HTS approaches have been also used to analyse the mixing (alloying) tendency of all binary inter-metallic compounds, i.e. screening hundreds of possible structures per system as in the AFLOW HT framework [32].

4 Computational prediction of RE-free magnetic crystal phases One of the most important and difficult step in the HT computational technique for materials design is the prediction of new stable crystal phases. So far many methods have been developed for predicting crystal structure as the simulated annealing [39], basin hopping [40], ab-initio random structure search [41], metadynamics [42], particle swarm optimization [41], cluster expansion method [35,44] and adaptive genetic algorithm (AGA) [45]. Some of these methods have been implemented in available online codes like CALYPSO [46], based on the particle swarm optimization, XTALOPT [47] and USPEX [48], based on AGA. Recently, the cluster expansion method and AGA have been used to predict new RE-free magnetic crystal phases.

4.1 Cluster expansion method In this approach the energy of a binary alloy is written as a series of effective cluster interactions [44, 49], which can be calculated either analytically [50], using a least-squares technique $[51,52]$ or with a linear-programming method [53]. Recently, A. Seko et al. improved the conventional iterative algorithm of this method increasing its accuracy significantly [44]. R. Drautz et al. [52,54] used the cluster expansion method in the analysis of the structure and magnetism in FeCo alloys finding a dense sequence of ground states.

4.2 Adaptive genetic algorithm AGA are adaptive heuristic algorithms used to solve optimization problems based on evolutionary ideas of natural selection and generics. AGA belongs to a larger class of evolutionary algorithms (EA) that generates solutions to optimization problems using various evolutionary techniques, such as inheritance, selection, mutation and crossover. Evolution is considered as an iterative process, which starts with a random generation of individuals of a population (a generation). Individuals are candidate solutions to the optimization problem and within AGA the population is evolved toward better solutions. In the case of the structure predicting an individual is a randomly generated crystal structure (cell lattice parameters and ion position) and the solution is the ground state phase of the system at certain pressure $(\mathrm{P})$ and temperature $(\mathrm{T})$ conditions (the structure with the lowest enthalpy for $\mathrm{T}=0 \mathrm{~K})$.

Although the method employs random sampling, AGA are by no means random. Instead they make use of historical information to direct the search into region of better performance within the search space. Thus, for each generation the fitness of individuals is evaluated. The fitness is a set of criteria, which guaranty evolution towards better solutions. For our problem, we are looking for stable structures, which are, energetically, at the minima of the potential energy surface (PES). A suitable fitness parameter is, therefore, the enthalpy or, at equilibrium conditions, the ground state energy of the system. Here come into play the DFT calculation methods. In order to accurately estimate the energy of a generated structure, the system undergoes a structural optimization. The unit cell shape and atom positions are relaxed by allowing them to change until cell stress and forces acting on atoms become insignificant. 


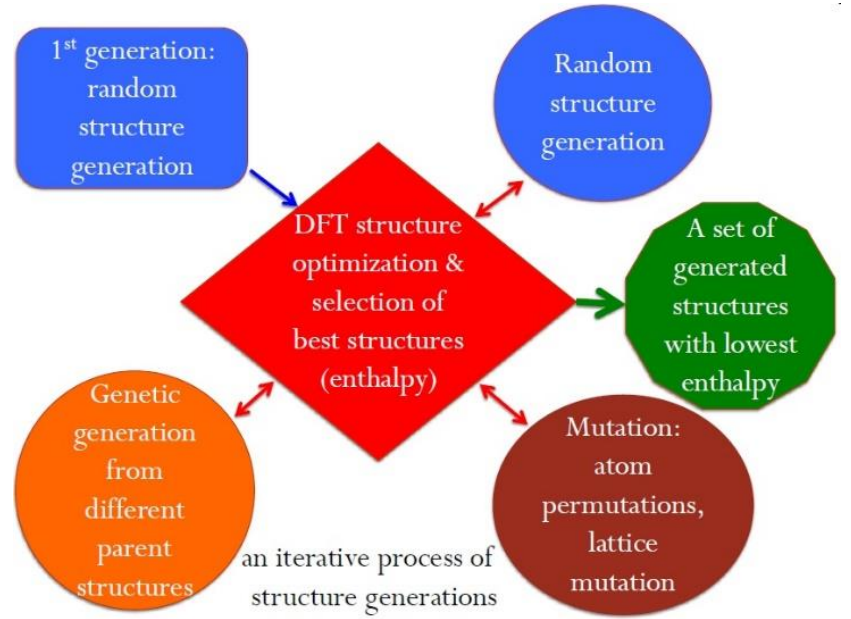

Figure 5 Diagram showing the structure prediction calculations through AGA approach.

The most fitted individuals are selected and subjected to genetic processes in order to form a new generation. The evolutionary approach applies three basic operators: selection, crossover, and mutation. The selection preserves the best fitted individuals, allowing them to pass into next generation. The crossover generates new individuals by recombination mechanism. A set of "good" structures are selected from current generation, and from this set pairs of "parent" structures are selected to produce a "child" solution. Slicing parent structures with subsequent recombination of different parts can generate a new structure. Finally, mutation is applied to introduce random structure modifications, in order to maintain diversity and inhibit the premature convergence. Swapping a pair of lattice vectors or permutating a pair of atoms will produce a structural mutation. Fig. 5 shows a diagram of the structure prediction calculations using genetic operations. Apart from genetic operations (see Fig. 6) the diversity of a population can be maintained by adding new randomly generated individuals. The number of minima of a PES increases exponentially with the number of atoms in the system, thus a continuous random sampling may ensure that various regions of the PES are probed. The new generation of candidate solutions is then used for the next iterative process, and algorithm stops when the maximum number of generation has been produced or the target fitness level has been reached.

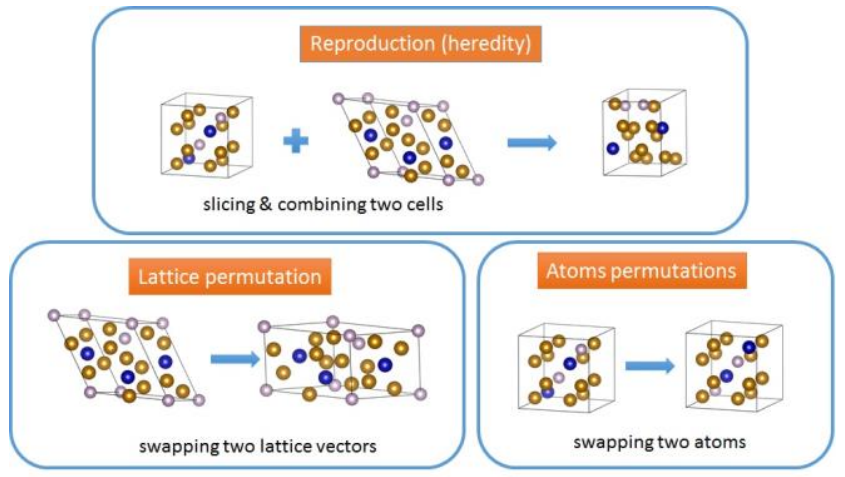

Figure 6 Schematic of genetic operations in AGA.

AGA approach has been recently used to understand and search for new magnetic materials. For example, a genetic algorithm has been applied to $\mathrm{Fe}_{1-\mathrm{x}} \mathrm{Co}_{\mathrm{x}}$ alloys by Nguyen and co-workers, finding that these alloys are highly configurationally degenerate with many additional offstoichiometric stable structures to the well-known B2 structure [55]. Moreover, AGA has been applied to $\mathrm{Zr}_{2} \mathrm{Co}_{11}$ polymorphs, a promising RE-free PM, identifying its hard crystal phase and explaining the physical origin of its high coercivity [56]. In the following, we present a software implementation of a particular AGA, which has been proposed for discovering new RE-free magnetic crystal phases in the context of the EU-H2020 NOVAMAG project [57]. Such a methodology has been preliminary compared to well-known experimentally reported properties of the $\mathrm{Fe}_{3} \mathrm{Sn}$ compound, showing an excellent agreement.

4.2.1 Validation of AGA as an efficient tool for exploring magnetic crystal structures: the case of $\mathrm{Fe}_{3} \mathrm{Sn}$ In literature one finds that several magnetic $\mathrm{Fe}-\mathrm{Sn}$ intermetallic compounds have been widely investigated experimentally by means of Mössbauer spectroscopy [58], X-ray diffraction [59], neutron scattering [60] and perturbed angular correlation [61]. Here, in order to show the capability of AGA for predicting magnetic crystal phases, we calculated the ground state and few low-energy metastable structures of the compound $\mathrm{Fe}_{3} \mathrm{Sn}$ (at temperature $\mathrm{T}=0 \mathrm{~K}$ and pressure $\mathrm{P}=0 \mathrm{~Pa}$ ) using AGA and we compared them with available experimental results.

Here AGA was implemented through the software USPEX [19] combined with the DFT code VASP $[62,63,64]$. Structure relaxation is performed in several stages. Since the generated structure, is, usually, far from equilibrium, a crude optimization is performed in the beginning to save computational time. Accuracy is gradually increased for subsequent runs. USPEX developers provide a procedure to perform a several-steps relaxation. It is a combination of ion positions and cell shape optimization, followed by a cell volume relaxation, which is repeated several times with increasing the accuracy of calculations. 
Increasing the accuracy means increasing the number of $\mathrm{k}$ points in the reciprocal space, and the tolerance of the electronic and ionic convergence. After some tests we have modified this procedure for the case of spin-polarized calculations. Thus, the first crude cell shape and ion position optimization is done for a non-magnetic system. Magnetic interactions are switch on at the second stage when a general relaxation (including the volume) is performed. At this stage the cut-off energy is increased by $40 \%$ with respect to the default VASP value. At the third stage we perform a set of volume calculations around the equilibrium volume. At each volume point cell shape and ion positions are optimized with a cut-off energy increased by $20 \%$. The equilibrium volume and energy are estimated from fitting $\mathrm{E}=\mathrm{E}(\mathrm{V})$ to 3rd order Birch-Murnaghan equation of state (EOS)

Once the fitness is evaluated, which in our case is the ground state energy of the system, USPEX applies a series of genetic operations to $65 \%$ of structures in order to create the next generation. $50 \%$ of all structures are generated by crossover, another by random generation (20\%), and mutation (30\%, lattice and atom permutations). Calculations continue until convergence criterion or the maximum number of generations is reached. A convergence criterion is, usually, a set of best structures persistent over several generations. Calculation time and required computational resources depend on the size of the system. For a 4 atom system, the size of the generation is about 10 , and the solution is achieved within 10 generations (about 100 structure relaxations). On 4 cores one USPEX run can be done in 23 days on a desktop computer. For a $\mathrm{N}=8$ atom system the size of generation is twice larger, and the convergence may require more than 20 generations, so it scales at least as $\mathrm{N}^{2}$, and the computational time and resources as $\mathrm{N}^{3}$. Thus, on single machine a run may take few weeks.

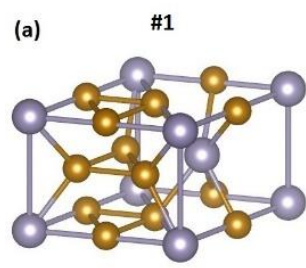

(c)

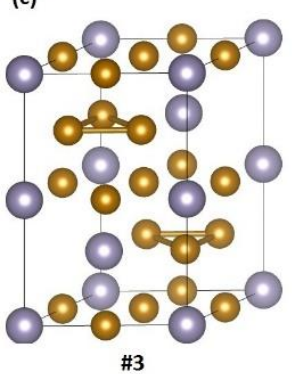

(b)

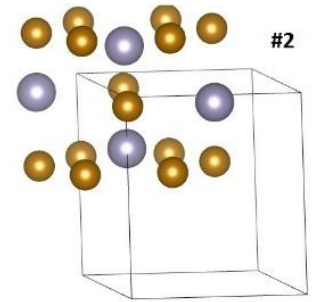

(d)

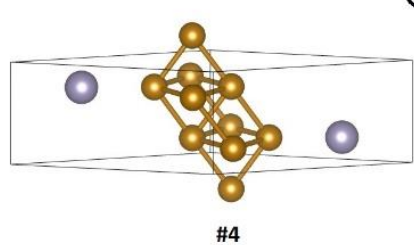

Following this procedure, we performed 3 simulations with 4,8 and 16 atoms/cell starting from scratch, where in all cases $\mathrm{Fe}$ and Sn concentrations were $75 \%$ and $25 \%$, respectively. In Fig. 7 we show the four structures with lowest energy found by USPEX+VASP, they are labelled as \#1 (Fig.6.a), \#2 (Fig.6.b), \#3 (Fig.6.c) and \#4 (Fig.6.d). Their equilibrium volume, energy, bulk modulus (B) and $B^{\prime}=d B / d P$ are estimated from fitting energy versus volume curve, $\mathrm{E}=\mathrm{E}(\mathrm{V})$, to 3rd order Birch-Murnaghan EOS. Figs. 8 show the energy differences $(\Delta \mathrm{E})$ versus unit cell volume and enthalpy differences $(\Delta \mathrm{H})$ versus pressure of these structures with respect to the most stable phase (structure \#1). We observe that the structure \#4 becomes the most stable one at moderate expansion $(\mathrm{P}=-5 \mathrm{~Pa})$. Some of their properties are shown in Table 3, where experimental data taken from Refs. [58,59] are also included. We see that the calculated symmetry group, unit cell volume, saturation magnetization and bulk modulus of the ground state (structure \#1) are in good agreement with the experimental data.

As a result, we can conclude that the high level of structure reproducibility showed by AGA suggests that this method could be an efficient way to explore unknown magnetic phases.
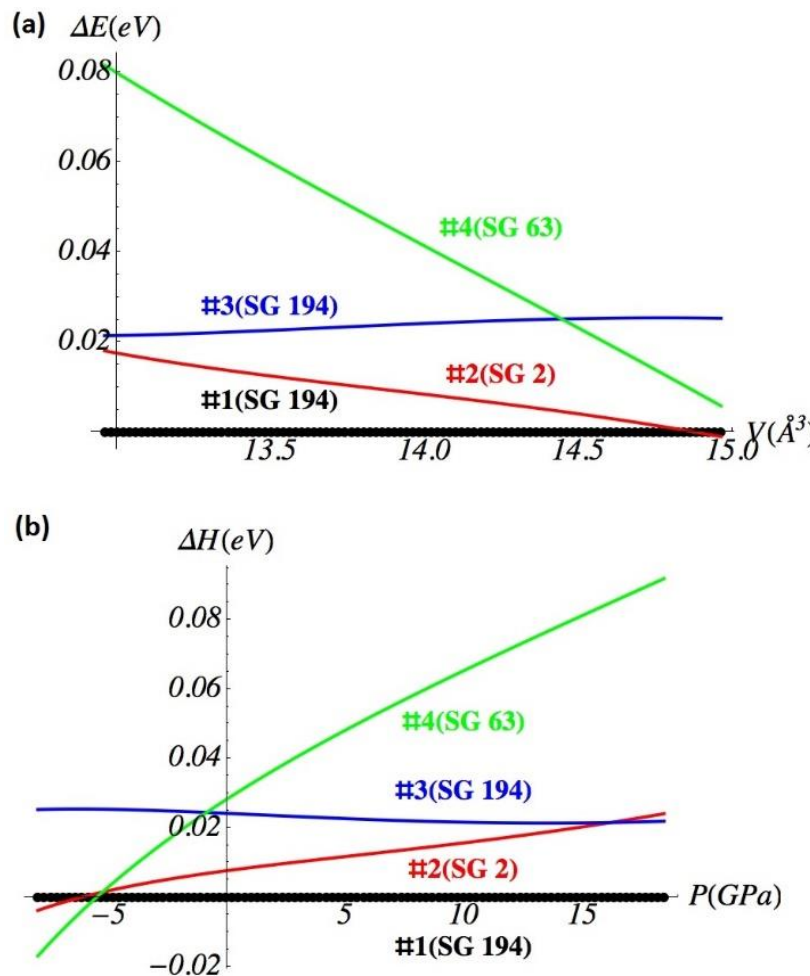

Figure 8 Energy differences $\Delta \mathrm{E}$ versus unit cell volume (a) and enthalpy differences $\Delta \mathrm{H}$ versus pressure (b) with respect to the most stable phase of $\mathrm{Fe}_{3} \mathrm{Sn}$ (structure \#1).

Figure 7 Structures calculated by AGA for $\mathrm{Fe}_{3} \mathrm{Sn}$. 
Table 3 Properties of $\mathrm{Fe}_{3} \mathrm{Sn}$ structures showed in Fig. 6.

\begin{tabular}{lccccccc}
\hline Fe 3 Sn & $\begin{array}{r}\text { Space } \\
\text { Group }\end{array}$ & $\begin{array}{c}E_{\text {cell }} / \text { atom } \\
(\mathrm{eV} / \text { atom })\end{array}$ & $\begin{array}{c}\mathrm{V}_{\text {cell }} / \text { atom } \\
\left(\mathrm{A}^{3} / \text { ion }\right)\end{array}$ & $\begin{array}{c}\mu_{0} \mathrm{M}_{\mathrm{S}} \\
(\mathrm{T})\end{array}$ & $\begin{array}{c}\mathrm{B} \\
(\mathrm{GPa})\end{array}$ & $\mathrm{B}^{\prime}$ \\
\hline Exp.[58,59] & 194 & - & 14.0625 & 1.41 & 122.0 & 5.3 \\
USPEX\#1 & 194 & -7.11868 & 14.0238 & 1.47 & 131.8 & 3.1 \\
USPEX\#2 & 2 & -7.11104 & 14.1699 & 1.37 & 121.6 & 5.4 \\
USPEX\#3 & 194 & -7.09452 & 13.9751 & 1.49 & 129.3 & 4.6 \\
USPEX\#4 & 63 & -7.09042 & 14.7286 & 1.35 & 105.4 & 5.0
\end{tabular}

5 Conclusions This paper has given an overview of the problem about the dependence on critical raw materials by PM technology. Discovering new RE-free magnetic phases is an urgent problem in different industrial value chains in the European Union. We discussed about the possibility of using HT computational techniques for materials design and discovery, and how such approach could be very useful for developing high performance RE-free PM. Finally, we present our approach relying on AGA for the discovery of new magnetic phases. We have preliminary validated this new tool, by applying AGA to understand experimental data on the $\mathrm{Fe}_{0.75} \mathrm{Sn}_{0.25}$ compound, finding an excellent agreement. In addition, we have found possible new theoretical low-energy magnetic metastable structures.

Our preliminary results ratify this methodology as an open opportunity to explore magnetic materials and suggest that with further improvements and effort, this approach could be used to provide initial RE-free crystal phases as input structures for a HT screening, where those structures with better magnetic properties for a PM may be selected and analysed in more detail.

Acknowledgements Authors acknowledge support from NOVAMAG project, under Grant Agreement No. 686056, EU Horizon 2020 Framework Programme for Research and Innovation (2014-2020).

\section{References}

[1] R. Skomski, J.M.D. Coey, "Permanent Magnetism," Institute of Physics, Bristol 1999.

[2] O. Gutfleisch, M. Willard, E. Bruck, C. Chen, S. Sankar, and J. Liu, Advanced Materials 23, 821-842 (2011).

[3] J.M.D. Coey "Magnetism and magnetic materials", Cambridge University Press, New York 2009.
[4] H. Zeng, R. Skomski, L. Menon, L. Y. Liu, S. Bandyopadhyay, D.J. Sellmyer, Phys. Rev. B 6, 134426 (2002).

[5] Lin Zhou, M.K. Miller, Ping Lu, Liqin Ke, R. Skomski, H. Dillon, Q. Xing, A. Palasyuk, M.R. McCartney, D.J. Smith, S. Constantinides, R.W. McCallum, I.E. Anderson, V. Antropov, M.J. Kramer, Acta Materailia 74, 224 (2014).

[6] R.W. McCallum, L.H. Lewis, R. Skomsky, M.J. Kramer, and I.E. Anderson, Annu. Rev. Mater. Res. 44, 451 (2014).

[7] K. Bourzac, Technol. Rev. 114, 58 (2011).

[8] Da Li, De Sheng Pan, Shao Jie Li, and Zhi Dong Zhang, Sci. China-Phys. Mech. Astron., 59, 617501 (2016).

[9] F. Jimenez-Villacorta, L. H. Lewis, "Nanomagnetism", OCP, Chap. 7, 2004.

[10] A. Brumme, "Wind energy deployment and the relevance of Rare-Earth", Springer Glaber 2014.

[11] U.S. Department of Energy, Critical Materials Strategy, December 2011.

[12] U.S. Geological Survey, Mineral Commodity Summaries, January 2015.

[13] http://www.mineralprices.com

[14] J.H. Rademaker, R. Kleijn, Y. Yang, J. Environ. Sci. Technol. 47, 10129 (2013).

[15] B. Sprecher, R. Kleijn, G.J. Kramer, J. Environ. Sci. Technol. 48, 9506 (2014).

[16] A. Walton, Han Yi, N.A. Rowson, J.D. Speight, V.S.J. Mann, R.S. Sheridan, A. Bradshaw, I.R. Harris, A.J. Williams, Journal of Cleaner Production, 104, 236 (2015).

[17] Kenji Baba, Yuzo Hiroshige, Takeshi Nemoto, Hitachi Review, 62, 8 (2013).

[18] J. Hafner, C. Wolverton, G. Ceder, MRS bulletin, 659, 31 (2006).

[19] R.M. Martin, "Electronic Structure Theory: Basic Theory and Practical Methods" (Cambridge University Press, Cambridge, UK, 2004).

[20] J. Kubler, "Theory of itinerant electron magnetism", (Oxford science publications, New York, 2000).

[21] G. Bihlmayer, Handbook of Magnetism and Advanced Magnetic Materials, John Wiley \& Sons, Vol.1, 2007.

[22] R. Wu, Handbook of Magnetism and Advanced Mag-netic Materials, John Wiley \& Sons, Vol.1, 2007.

[23] A. I. Liechtenstein, M. I. Katsnelson, and V. A. Gubanov, J. Phys. F: Metal Phys. 14, L125 (1984).

[24] P. Bruno, Phys. Rev. Lett. 90087205 (2003).

[25] Rosengaard, N.M. and Johansson, B., Phys. Rev. B, 55, 14975-14986 (1997).

[26] Antropov, V.P., Katsnelson, M.I., van Schilfgaarde, M. and Harmon, B.N., Phys. Rev. Lett., 75, 729-732 (1995).

[27] I. Turek , J. Kudrnovský , V. Drchal \& P. Bruno, Philosophical Magazine, 86:12, 1713-1752 (2006).

[28] R. F. L. Evans, W. J. Fan, P. Chureemart, T. A. Ostler, M. O. A. Ellis and R. W. Chantrell, J. Phys.: Condens. Matter 26, 103202 (2014).

[29]N.Kazantseva, D.Hinzke, U.Nowak, R.W.Chantrell, U.Atxitia and O.Chubykalo-Fesenko, Phys Rev B 77 (2008) 184428.

[30] D. Hinzke, U. Atxitia, K. Carva, P. Nieves, O. ChubykaloFesenko, P. M. Oppeneer, and U. Nowak, Phys. Rev. B, 92, 054412 (2015). 
[31] S. Curtarolo, G. L. W. Hart, M. Buongiorno-Nardelli, N. Mingo, S. Sanvito, O. Levy., Nature Mater. 12, 191 (2013).

[32] S. Curtarolo, W. Setyawan, S. Wang, J. Xue, K. Yang, R. H. Taylor, L. J. Nelson, G. L.W. Hart, S. Sanvito, M. Buongiorno-Nardelli, N. Mingo, O. Levy, Comp. Mater. Sci. 58, 227 (2012).

[33] A. Jain, G. Hautier, C. J. Moore, S. Ping Ong, C. C. Fischer, T. Mueller, K. A. Persson, G. Ceder., Comp. Mater. Sci. 50, 2295 (2011).

[34] A. R. Oganov and C. W. Glass., J. Chem. Phys. 124, 244704 (2006).

[35] G. L. W. Hart, V. Blum, M. J. Walorski, A. Zunger, Nature Mater. 4, 391 (2005).

[36] D. Morgan, J. Rodgers and G. Ceder, J. Phys. Condens. Matter 15, 4361 (2003).

[37] C. Ortiz, O. Eriksson, M. Klintenberg, Comput. Mater. Sci. 44, 1042-1049; (2008).

[38] Gilleßen, M. and Dronskowski, R. Comput. Chem. 31(3):612-9; (2013).

[39] S. Kirkpatrick, C.D. Jr Gelatt and M. P. Vecchi, Science 220 671-80 (1983).

[40] D. Wales and J. Doye, J.Phys.Chem. A 101 5111-6 (1997).

[41] C. J. Pickard and R. J. Needs, J. Phys.: Condens. Matter 23, 053201 (2011).

[42] P. Raiteri, R. Martonak, M. Parrinello, Angew. Chem. Int Edit. 44, 3769 (2005).

[43] Y. Wang, J. Lv, L. Zhu and Y. Ma, Phys. Rev. B 82, 094116 (2010).

[44] A. Seko, Y. Koyama, and I. Tanaka, Phys. Rev. B 80, 165122 (2009)

[45] M. Ji, K. Umemoto, C. Z. Wang, K. M. Ho and R. M. Wentzcovitch, Phys. Rev. B 84, 220105 (2011).

[46] Y. Wang, J. Lv, L. Zhu, Y. Ma, Comput. Phys. Commun.183 2063-70 (2012).

[47] David C. Lonie, Eva Zurek, Computer Physics Communications 182, 372-387 (2011).

[48] Andriy O. Lyakhov, Artem R. Oganov, Harold T. Stokes, Qiang Zhu, Computer Physics Communications 184, 11721182 (2013).

[49] J. M. Sanchez, F. Ducastelle, and D. Gratias, Physica A 128, 334 (1984).

[50] J. W. D. Connolly and A. R. Williams, Phys. Rev. B 27, 5169 (1983).

[51] Z. W. Lu, S.-H. Wei, A. Zunger, S. Frota-Pessoa, and L. G. Ferreira, Phys. Rev. B 44, 512 (1991).

[52] A. Díaz-Ortiz, R. Drautz, M. Fahnle, H. Dosch, and J. M. Sanchez, Phys. Rev. B 73, 224208 (2006).

[53] G. D. Garbulsky and G. Ceder, Phys. Rev. B 51, 67 (1995).

[54] R. Drautz, A. Díaz-Ortiz, M. Fahnle, and H. Dosch, Phys. Rev. Lett. 93, 067202 (2004).

[55] M. C. Nguyen, X. Zhao, M. Ji, Cai-Zhuang Wang, B. Harmon, Kai-Ming Ho, J. App. Phys. 111, 07E338 (2012).

[56] X. Zhao, M. C. Nguyen, W. Y. Zhang, C. Z. Wang, M. J. Kramer, D. J. Sellmyer, X. Z. Li, F. Zhang, L. Q. Ke, V. P. Antropov, K. M. Ho, Phys. Rew. Lett. 112, 045502 (2014).

[57] H2020-NMBP23 2015-NOVAMAG "Novel, critical materials free, high anisotropy phases for permanent magnts, by design" Grant Agreement No. 686056.

[58] G. Trumpy, E. Both, C. Dje'ga-Mariadassou, P. Lecocq, Phys. Rev. B 2, 3477 (1970).

[59] H. Giefers, M. Nicol, Journal of Physics and Chemistry of Solids 67, 2027-2032 (2006).
[60] G. Venturini, D. Fruchart, J. Hübsch, G. LeCae“ r, B. Malaman, B. Roques, J. Phys. F 15 (1985) 427.

[61] B. Wodniecka, P. Wodniecki, A. Kulinska, A.Z. Hrynkiewicz, J. Alloys Compd. 321 (2001) 1.

[62] G. Kresse and J. Hafner, Phys. Rev. B 47, 558 (1993); ibid. 49, 14251 (1994).

[63] G. Kresse and J. Furthmuller, Comput. Mat. Sci. 6 , 15 (1996).

[64] G. Kresse and J. Furthmuller, Phys. Rev. B 54 , 11169 (1996). 